\title{
EFECTO DEL SILICIO Y PLAGUICIDAS EN LA FERTILIDAD DEL SUELO Y RENDIMIENTO DEL ARROZ ${ }^{1}$
}

\author{
Parménides Furcal-Beriguete ${ }^{2}$, Alejandra Herrera-Barrantes ${ }^{3}$
}

\section{RESUMEN}

Efecto del silicio y plaguicidas en la fertilidad del suelo y rendimiento del arroz. El objetivo de este trabajo fue evaluar el efecto del silicio en la fertilidad del suelo, la incidencia de enfermedades y plagas insectiles, el rendimiento y la calidad de granos del cultivo de arroz. El estudio se realizó en La Vega, Florencia, San Carlos, Costa Rica entre mayo y octubre en los años 2010 y 2011, en el mismo lote con la variedad CR 4477. Se establecieron cinco tratamientos: silicio al suelo, silicio al suelo más plaguicidas (alternativas químicas), silicio foliar, silicio foliar más plaguicidas y testigo comercial. Como fuente se utilizaron silicio en polvo al 70\% de $\mathrm{SiO}_{2}$, aplicado quince días antes de siembra en dosis de $100 \mathrm{~kg} \mathrm{SiO}_{2} /$ ha, y líquido concentrado $40 \% \mathrm{SiO}_{2}$ y $36 \% \mathrm{MgO}$, aplicado al follaje en dosis de $4 \mathrm{l} / \mathrm{ha}$ a los 17 y 30 días después de la siembra. El suelo del orden inceptisol de formación aluvial, al inicio del experimento tenía $\mathrm{pH} 4,9$, valores de $\mathrm{P}$ y Si disponibles de 29 ppm y 44,7 ppm, respectivamente, acidez intercambiable $1,2 \mathrm{cmol}(+) / 1$ y suma de bases $19,11 \mathrm{cmol}(+) / 1$. No hubo diferencia estadística significativa del silicio en la fertilidad del suelo, la incidencia de plagas y enfermedades, y en la calidad molinera; sin embargo, la combinación del silicio aplicado al suelo y el uso de plaguicidas incrementaron el contenido de zinc y cobre en el suelo, y del zinc y magnesio en las hojas de arroz, pero este efecto no se tradujo en rendimiento y calidad de granos. La aplicación de plaguicidas influyó positivamente en el peso y rendimiento del arroz en granza $(\mathrm{p} \leq 0,05)$.

Palabras claves: Oryza sativa, acidez de suelo, silicio aplicado al suelo, zinc en hojas de arroz.

\begin{abstract}
Effect of silicon and pesticides in soil fertility and rice yield. The objective of this work was to evaluate the effect of silicon on the soil fertility, the incidence of diseases and insect pests, the yield and quality of rice grains, variety CR 4477. The study was conducted in La Vega, Florencia, San Carlos, Costa Rica between May and Octuber in 2010 and 2011 in the same field. Five treatments were established: silicon to soil, silicon to soil plus pesticides (chemical alternatives), silicon to foliage, silicon to foliage plus pesticides and a commercial control. Sources were silicon powder at $70 \%$ of $\mathrm{SiO}_{2}$ applied 15 days before sowing at a dose of 100 $\mathrm{kg} \mathrm{SiO}_{2} / \mathrm{ha}$ and $40 \% \mathrm{SiO}_{2}$ and $36 \% \mathrm{MgO}$ concentrated liquid applied to the foliage at a dose of 4 1/ha at 17 and 30 days after planting. The soil was an alluvial formation inceptisol order, and at the beginning of the experiment had a $\mathrm{pH}$ of $4.9, \mathrm{P}$ and $\mathrm{Si}$ values of 29 and $44.7 \mathrm{ppm}$ respectively, exchangeable acidity $1.2 \mathrm{cmol}(+) / 1$ and base sum $19.11 \mathrm{cmol}(+) / \mathrm{l}$. The results showed no effect of silicon in soil fertility, the incidence of pests and diseases, and milling quality, except that the combination of silicon applied at soil and the pesticides used increased the zinc and copper content in the soil, also the zinc and magnesium in the leaves of rice, but this effect did not result in yield and grain quality. Results also suggest that the use of pesticides positively influenced weight and paddy yield $(\mathrm{p} \leq 0.05)$.
\end{abstract}

Keywords: Oryza sativa, soil acidity, silicon applied to soil, zinc in rice leaves.

\footnotetext{
Recibido: 21 de febrero, 2013. Aceptado: 28 de octubre, 2013. Este trabajo es un resumen del proyecto "Efecto del silicio en la fertilidad de suelo, incidencia de enfermedades y el rendimiento del cultivo de arroz" código 5402-2151-8601, financiado por la Vicerrectoría de Investigación y Extensión del Instituto Tecnológico de Costa Rica (ITCR).

2 Escuela de Ingeniería en Agronomía, Instituto Tecnológico de Costa Rica (ITCR). Sede San Carlos, Costa Rica. Apartado: 223-21001, Alajuela, C. Quesada.pafurcal@itcr.ac.cr, pfurcal@hotmail.com (autor para correspondencia).

3 Laboratorios Doctor Obregón. Residencial Jerez, \#49, 800m norte de Global Park, La Aurora Heredia. aherrera@ doctor-obregon.com
} 


\section{INTRODUCCIÓN}

El arroz es el alimento básico para más de la mitad de la población mundial, además es el cultivo más importante del mundo cuando se considera la superficie en que se cultiva. Aunque es ampliamente sembrado en el continente americano, no alcanza los niveles de producción y empleo para la población rural como se produce en la mayor parte de Asia (Infoagro 2010).

El arroz como grano básico para la alimentación humana, tiene una alta repercusión en la actividad agrícola de Centro América y el Caribe, en el caso específico de Costa Rica, el consumo per cápita es de $50,98 \mathrm{~kg}$ pilado por año de este cereal, además ha tenido gran auge tanto en su expansión como en el desarrollo de nuevas tecnologías productivas, que se ven reflejadas en el aumento de la producción, el rendimiento o la reducción de los costos. Prueba de ello, se puede mencionar que en el periodo 2009-2010, nuestro país tuvo una siembra de 66415 hectáreas de arroz y para 2011-2012 la siembra fue de 77240 ha, para una producción nacional de 250849 y 261644 toneladas de arroz granza seca y limpia, respectivamente. Esta actividad tiene un alcance de 1356 productores y quince agroindustrias (CONARROZ 2013).

En Costa Rica, al momento de establecer este estudio y en la actualidad, se distribuyen fuentes de silicio importadas. Estos productos son recomendados para cualquier especie de cultivo, sin considerar las características de suelos donde se encuentran plantados y en muchos casos sin estudios respecto a dosis y frecuencia con que se deben aplicar.

Para Costa Rica existen pocos resultados publicados de investigaciones que respalden los beneficios que pueden generar las fuentes de silicio, ya sea al suelo, a los cultivos en los cuales se recomiendan; tampoco hay información referente al costo/beneficio que el productor puede obtener. En ese sentido, se recurre a investigaciones producidas fuera de nuestras fronteras, donde se encuentra que, el uso de estos productos tienen bondades cuando son utilizados en gramíneas y en suelos tropicales, principalmente cuando estos tienen tendencia ácida o con otra degradación de fertilidad química (Fihlo et al. 2000, Hernández 2002, Chaudhary et al. 2003, Quero 2008, Viana 2008b).

Para lograr buenos niveles de silicio asimilable por las plantas, deben aplicarse al suelo productos que al reaccionar con el agua formen ácido silícico, que es débilmente adherido en el suelo. Este, a pesar de tener poca migración en el suelo (Fertilizante de Centroamérica S.A 2004), tiene el inconveniente que puede perderse por lixiviación (Viana 2008b). Pero además, los silicatos tienen otros efectos importantes como los son la transferencia de resistencia contra enfermedades, ataque de insectos y al "volcamiento" de las plantas de arroz (Fihlo et al. 2000, Hernández 2002, Quero 2007).

Estos estudios se han llevado a cabo mayoritariamente en arroz y caña de azúcar (Fihlo et al. 2000, Hernández 2002, Kornörfer et al. 2002, Chaudhary et al. 2003). De ese modo, Chaudhary et al. (2003) indican que una buena producción de arroz puede extraer del suelo entre 467 y $560 \mathrm{~kg} / \mathrm{ha}$ de $\mathrm{Si}$, incrementándose la producción y acumulación de $\mathrm{SiO}_{2}$ en la epidermis de las hojas. Winslow (1992) y Datnoff et al. (1997), mencionan que el $\mathrm{Si}$ se acumula en forma activa en arroz en concentraciones iguales o mayores al 5\%, esto le confiere a la planta no solo resistencia a enfermedades fúngicas sino también promueven un mayor crecimiento. El silicio se absorbe como ortosilícico $\mathrm{Si}(\mathrm{OH})_{4}$ y metasilícico $\left(\mathrm{H}_{2} \mathrm{SiO}_{3}\right)$, en arroz del 4 a $7 \%$ de la materia seca aérea es silicio, mientras que en otras gramíneas es de 1 a $2 \%$ (Wild 1992).

En el caso de arroz, se ha comprobado que el silicio induce una excelente resistencia contra enfermedades, producto de Rhizoctonia, Pyricularia, Helminthosporium, Rynchosporium, Sarocladium, etc. (Viana 2008b), las cuales, según la variedad y la época de siembra son muy importantes en la producción de arroz en Costa Rica.

En el país, el hongo Sarocladium oryzae en conjunto con las bacterias Pseudomonas y Xanthomonas y el ácaro Steneotarsonemus spinky forman un complejo que ocasiona, alto índice de vaneo y manchado del grano ${ }^{4}$. En ese sentido, el efecto de $S$. oryzae ha sido una de las enfermedades más discutidas en los últimos años y que las esporas de este hongo son transportadas por S. spinky, causando la pudrición de la vaina y manchado del grano. Este complejo fue reportado en Costa Rica en mayo del 2004; desde entonces los esfuerzos se han enfocado en revisar la información científica y en capacitación con la finalidad de desarrollar investigaciones básicas y

Rivera, C. 2009. Causas del vaneo y manchado del grano de arroz. El Pelón de la Bajura, Liberia. Comunicación personal. 
aplicadas que permitan el manejo del complejo ácarohongo (Almaguel y Botta 2005).

Los suelos tropicales normalmente son ácidos o con tendencia a la acidez, debido a la baja saturación de bases, dada por la extracción de las plantas por el uso intensivo de los suelos, la lixiviación y la escorrentía a causa de las lluvias a través del tiempo, al material parental, a procesos de nitrificación, descomposición de la materia orgánica o bien a la baja capacidad de intercambio catiónica por su origen, es decir, baja fertilidad. La presencia de suelos degradados es común en América Latina y, Costa Rica no es la excepción.

Los beneficios que pueden ser alcanzados con el aporte adecuado del silicio, es la restauración de la fertilidad de los suelos a través del tiempo, al elevar la capacidad de intercambio catiónica, mejorar el contenido de calcio, magnesio, fósforo, entre otros elementos (Quero 2008, Hernández 2002, Fihlo et al. 2000).

La elevación de la concentración del silicio, también tiene efecto en la disminución de la toxicidad por hierro (Fe) y manganeso ( $\mathrm{Mn}$ ) en el cultivo de arroz (Hernández 2002, Fihlo et al. 2000). La razón más acertada es que la presencia de silicio en las plantas, hace que en las hojas y tallos se incremente la cantidad de oxígeno, el cual es impulsado hacia el parénquima de las raíces, oxidando la rizosfera, y logrando que el Fe y el Mn reducido se oxide, evitando una excesiva toma de estos elementos por parte de las plantas (Viana 2008b). La toxicidad por Fe y Mn en arroz se produce en Costa Rica, debido al manejo del agua en área bajo riego o por encharcamiento en zonas donde se siembra este cultivo en secano.

Es probable que por estos beneficios, en Japón el $25 \%$ del área cultivada con arroz reciba anualmente aplicaciones de silicatos de calcio que puede variar entre 0,5 a 1,0 t/ha. De la misma manera, en Brasil desde 1990 la siembra de arroz en zonas degradadas recibe aporte de silicatos en cantidades recomendadas entre 1,5 a 2,0 t/ha (Fihlo et al. 2000).

Por lo anterior, se estableció un experimento con el uso de silicio en arroz, cuyo objetivo fue evaluar el efecto de la aplicación de silicio y alternativas químicas (plaguicidas) sobre la fertilidad del suelo, la incidencia de enfermedades y plagas insectiles, el rendimiento y la calidad de arroz.

\section{MATERIALES Y MÉTODOS}

\section{Localización del área experimental y siembra}

El estudio de campo comprendió dos períodos, desde abril a octubre de los años 2010 y 2011, en el mismo lote en un suelo inceptisol de origen aluvial en la finca La Vega, localizada en Florencia de San Carlos, Alajuela, Costa Rica. El lote se encuentra a una altura de $75 \mathrm{msnm}$, en las coordenadas geográfica $10^{\circ}$ 26' 22" N y $84^{\circ} 31^{\prime} 38^{\prime \prime}$ O. La precipitación durante cada uno de los períodos de campo fue de 2229,7 y 2144,6 mm. La temperatura media anual de la zona en los meses correspondientes al período del estudio fue de $26,2^{\circ} \mathrm{C}$, con máxima y mínima de $31^{\circ} \mathrm{C}$ y $21,4^{\circ} \mathrm{C}$, respectivamente.

La variedad utilizada fue CR 4477, a una densidad de siembra de $128 \mathrm{~kg} / \mathrm{ha}$, sembrada en secano en hilera con sembradora accionada por tractor. Esta variedad tradicionalmente se siembra en la zona, tiene la cualidad de presentar buena adaptación en secano y muy buena bajo riego. La planta de arroz presenta tres fases fenológicas claramente definidas, vegetativa, reproductiva y maduración. En esta variedad, la primera fase finaliza a los 51 días después de la germinación (DDG), cuando se inicia la diferenciación de la panícula, la fase reproductiva inicia al terminar la vegetativa y finaliza con el inicio de la formación de la panícula y la floración, en esta variedad se produce a los 80 DDG, finalmente, la fase de maduración comprende el período entre la floración y la maduración completa del grano, con el final de esta etapa se ha completado el ciclo de la planta, es indicativo del momento de la cosecha, que en este material genético se realiza a partir de los 115 DDG (ONS 2003).

El suelo presentó las siguientes características: $\mathrm{pH}$ 4,9, 29 ppm de P, 44,7 ppm de Si, acidez intercambiable y suma de bases de 1,2 y $19,11 \mathrm{cmol}(+) / 1$, respectivamente, y alto contenido de $\mathrm{Fe}(564 \mathrm{ppm})$. Al finalizar la cosecha se realizaron análisis de suelo, con las características mencionadas anteriormente, en cada una de las unidades experimentales en los dos años consecutivos, utilizando el método Olsen modificado para $\mathrm{P}, \mathrm{K}$ y elementos menores, $\mathrm{KCl} 1 \mathrm{M}$ para acidez, $\mathrm{Ca}$ y $\mathrm{Mg}$ y HOAC 0,5 M para el caso del Si. La toma de las muestras foliares se realizó a los 90 días después de la siembra 
(DDS) en los dos años (2010 y 2011), en la hoja más joven abierta, normalmente corresponde a la tercera hoja.

\section{Variables evaluadas}

Para el muestreo de incidencia de enfermedades y plagas insectiles se utilizó una cuadrícula de 0,25 $\mathrm{m}^{2}$, elaborada con tubo de PVC con diámetro de $1 / 2$ ", colocándola al azar en cada una de las unidades experimentales. En estas se hicieron las evaluaciones o conteo de presencia de signos y síntomas de enfermedades, larvas, pupas y adultos de plagas, cada dos semanas, se inició a los 55 DDS y finalizó a los 95 DDS, es decir a los 55, 67, 81 y 95 DDS. En el área de cada cuadrícula se hizo el conteo total de plantas y de presencia de enfermedades y plagas por planta, obteniendo de ese modo la incidencia de las enfermedades, es decir, en cada unidad experimental y fecha de conteo se relacionó el número de plantas afectadas con el número total de estas por cuadrícula, encontrándose Rhizoctonia sp. y en menor grado Helminthosporium sp. Para el caso de la presencia de insectos, en cada área de la cuadrícula por fecha de medición y por unidad experimental se hizo el conteo de presencia total de larvas, pupas y adultos. Además, durante la cosecha hecha a los 120 DDS, se tomaron muestras que fueron enviadas al laboratorio de la Corporación Nacional Arrocera (Reforma del Decreto N`34487MEIC-MAG-S RTCR 406-2007 2008), determinándose el porcentaje de granos manchados producto de la incidencia de enfermedades en los tratamientos.

La evaluación del rendimiento se realizó en un área de $6,4 \mathrm{~m}^{2}$ (1,6 $\mathrm{m}$ de ancho y $4 \mathrm{~m}$ de largo), área que corresponde a la cosecha de doce hileras de plantas de arroz en cada área útil por unidad experimental. De la cosecha se tomaron muestras entre 4 y $5 \mathrm{~kg}$ que fueron enviadas a CONARROZ para determinar la calidad molinera que incluye: impurezas, humedad, rendimiento de pilada, grano entero, quebrado grueso, puntilla, semolina, porcentaje de granos con cincuenta porciento o más de su superficie con apariencia al yeso, grano manchado, entre otros. También se evaluaron los componentes de rendimiento, como granos llenos, vanos, peso de 1000 granos. Además de lo anterior, se analizaron los tallos totales y efectivos (plantas con y sin panícula). La longitud de panícula se midió desde el nudo ciliar hasta el ápice de la misma y se clasificó en las categorías: categoría 1 : menor a $20 \mathrm{~cm}$, categoría 2 : entre 20 a $25 \mathrm{~cm}$ y categoría 3: mayores a $25 \mathrm{~cm}$.

\section{Tratamientos y diseño experimental}

Se establecieron cinco tratamientos: 1. Sin silicio pero con tratamiento químico para el control de enfermedades y plagas (tratamiento testigo $=\mathrm{TT}$ ), 2 . Con silicio aplicado al suelo y con tratamiento químico para el control de enfermedades y plagas (silicio al suelo más plaguicidas o alternativas químicas $=$ SSP), 3 . Con silicio aplicado al suelo y sin tratamiento químico para el control de enfermedades y plagas (tratamiento silicio al suelo $=$ SS), 4. Con silicio aplicado al follaje y con tratamiento químico para el control de enfermedades y plagas (silicio foliar más plaguicidas o alternativas químicas $=$ SFP), 5. Con silicio aplicado al follaje y sin tratamiento químico para el control de enfermedades y plagas (silicio foliar $=\mathrm{SF}$ ).

Cada tratamiento contó con cuatro repeticiones, cada una con una superficie de $300 \mathrm{~m}^{2}$. Las unidades experimentales, al igual que los bloques, se separaron por $0,5 \mathrm{~m}$. El ensayo se realizó en un diseño de bloques al azar con arreglo factorial aumentado $(2 \times 2+1)$, donde los factores son silicio, sea este aplicado al suelo o foliar, y aplicación o no de productos químicos para el control de plagas y enfermedades en cada uno de los tratamientos de silicio (suelo y foliar), más un adicional que fungió como testigo (TT). Para el análisis de los datos de cada variable se utilizó el programa InfoStat versión 2008 (Balzarini et al. 2008), primeramente se hizo un ANDEVA con y sin la inclusión del tratamiento testigo, posteriormente, en aquellas variables que hubo diferencias significativas, se procedió a realizar la prueba de Tukey para el primer caso (con todos los tratamientos SS, SSP, SF, SFP y TT) y para el caso sin la inclusión del testigo, a los tratamientos silicio con y sin plaguicidas (SS, SSP, SF, SFP).

El tratamiento al suelo consistió en un polvo fino a base de silicio aplicado al voleo 15 días antes de la siembra en una dosis de $100 \mathrm{~kg} / \mathrm{ha}$ de $\mathrm{SiO}_{2}$, en las mismas unidades experimentales en los dos años consecutivos. Mientras que en el tratamiento con silicio foliar se utilizó un producto comercial formulado como suspensión concentrada con $40 \% \mathrm{SiO}_{2}$ y $36 \% \mathrm{MgO}$ sin contaminantes con otros minerales, aplicado al follaje sin ser mezclado con otros productos, con bomba manual a la dosis de 4 1/ha por aplicación, a los 17 y 30 días después de la siembra, este tratamiento, igual que los demás, se hizo en las mismas parcelas en los dos años consecutivos. 
El tratamiento con alternativas químicas (plaguicidas), únicamente se utilizó como testigo, con el control químico habitual de la finca, aplicado con un equipo con catorce boquillas, accionado por la toma de fuerza de un tractor.

Los productos usados en el área experimental consistieron en herbicidas, insecticidas, fungicidas y fertilizantes, como se indica a continuación.

Herbicidas antes de la germinación del cultivo Cyclosulfamurón 70 DG (Sulfamoilurea) $47 \mathrm{~g} /$ ha, Pendimentalina 50 EC (Dinitroanilina) 2 1/ha y Butaclor-cloroacetamida 60 EC 3 1/ha. A los 45 DDS se aplicó Propanil-anílofos 48 EC (3,4-dicloropropionanilida) 4 1/ha, Bispiribac sodio 40,8 SC (Ácido pirimidiniloxibenzoico) $115 \mathrm{ml} / \mathrm{ha}$, Cyhalofop n-butil éster 18 EC Butil-2-[4-(4-ciano-2fluorofenoxi)fenoxi] 0,6 l/ha. A los 80 DDS se aplicó Pretilaclor 500 EC (2-cloro-2,6-dietil-N-(2-propoxietil) acetanilida) 1,8 1/ ha y Triclopir 48 EC $(3,5,6$-Tricloro-2 piridiloxy ácido acético (triclopir BEE)) 0,47 l/ha. A nivel comercial en lotes con siembra en suelos mínima labranza se aplicaron, además Glifosato 35,6 SL (N-fósfono-metil glicina) y 2,4-D Amina 2-(2,4-diclorofenoxi) ácido acético.

Los insecticidas y fungicidas fueron: Dimetoato 48 EC (Órganofosforado) 0,5 1/ha y Carbendazina $50 \mathrm{SC}$ (Metil benzimidazol-2-ylcarbamato) 0,6 1/ha, a los 20 DDS. Lambda-cithalotrina 5 CS (Piretroide) $0,35 \mathrm{l} / \mathrm{ha}$ y terbuconazole-triadimenol $30 \mathrm{EC}(0,5 \mathrm{l} /$ ha), a los 45 DDS. Cypermetrina- dimetoato $25 \mathrm{EC}$ (Poretroide-órganofosforado) 1 1/ha y Carbendazina 50 SC (Metil benzimidazol-2-ylcarbamato) 1 l/ha más Valimidamicina A. 10 SL (Antibiótico) 0,8 1/ha, a los 80 DDS. Finalmente a los 93 DDS se aplicaron el insecticida Clorpyrifos 5 GR a la dosis de $10 \mathrm{~kg} / \mathrm{ha}$ y los fungicidas Azoxystrobin-metoxiacrilato 50 WG 0,3 1/ha y Carbendazina 50 SC (Metil benzimidazol2-ylcarbamato) 1 1/ha.

El plan de fertilización al suelo consistió en la aplicación de las fórmulas 10-30-10 (125 kg/ha) y 13-24-20 (135 kg/ha) durante la siembra de 2010 y 2011 respectivamente; $26-0-26(150 \mathrm{~kg} / \mathrm{ha})$ y $30-$ $0-15-0-0-4(\mathrm{~S})(150 \mathrm{~kg} / \mathrm{ha})$ a los 25 DDS durante la siembra de 2010 y 2011 respectivamente. Finalmente, a los 60 DDS las fórmulas 46-0-0 y 18-0-30-0-0-1,05 (Zn) $(150 \mathrm{~kg} / \mathrm{ha}$ cada una $)$ en el orden de la siembra. A nivel del follaje se aplicaron fertilizantes foliares a los 45 DDS. Los productos usados fueron dos multiminerales con elementos mayores más $\mathrm{CaO}$ 1,4\% y extractos de plantas $70 \%$ el primero y el segundo nitrógeno total $7,2 \%$, aminoácidos $26 \%$ y vitaminas, a la dosis de 1,0 y 0,75 1/ha, respectivamente. Luego a los 93 DDS se aplicó otro multimineral solución líquida (SL) (2,5 1/ha), que además de los elementos mayores contiene Zn: 664, Cu: 332, Mn: 332, Fe: 415, entre otros componentes como polisacáridos.

\section{RESULTADOS Y DISCUSIÓN}

\section{Efecto de la aplicación de silicio en la fertilidad de los suelos}

Los análisis de suelos mostraron que únicamente hubo diferencia estadística ( $\mathrm{p}$-valor $\leq 0,05)$ entre los tratamientos con respecto al elemento zinc y en las interacciones entre la aplicación de silicio y de plaguicidas en los elementos zinc y cobre disponibles en el suelo en la siembra de 2011 (Figuras 1, 2 y 3). Estos resultados contradicen lo expuesto por Quero y Cárdenas (2006), quienes indican que el silicio aplicado al suelo optimiza la nutrición en elementos como fósforo $(\mathrm{P})$, potasio $(\mathrm{K})$ y zinc $(\mathrm{Zn})$.

Los resultados obtenidos a partir del análisis de suelo hecho previo a la siembra y los realizados posterior a las cosechas fueron similares, sin presentarse efecto del silicio en las variables $\mathrm{pH}$, acidez intercambiable, sumatoria de bases, fósforo,

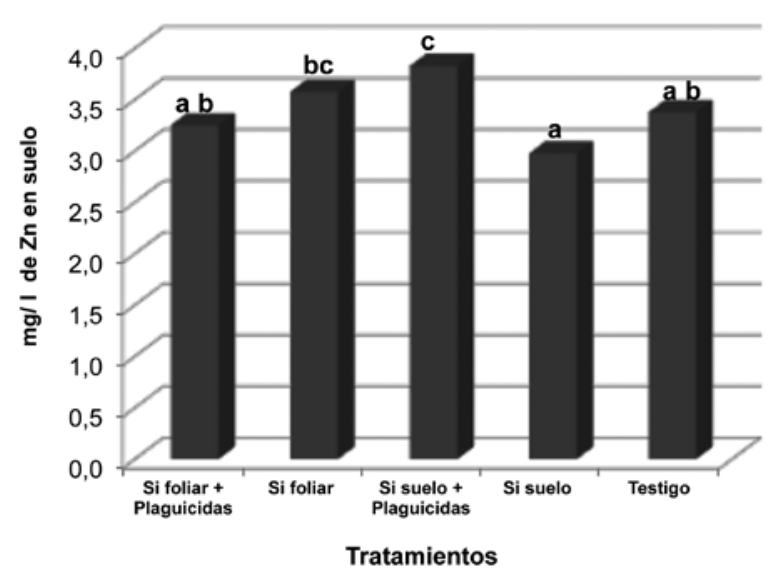

Figura 1. Representación de zinc en el suelo en la cosecha de arroz del año 2011. Santa Clara, San Carlos, Costa Rica. Octubre 2011. Letras diferentes indican que hubo diferencias significativas. 


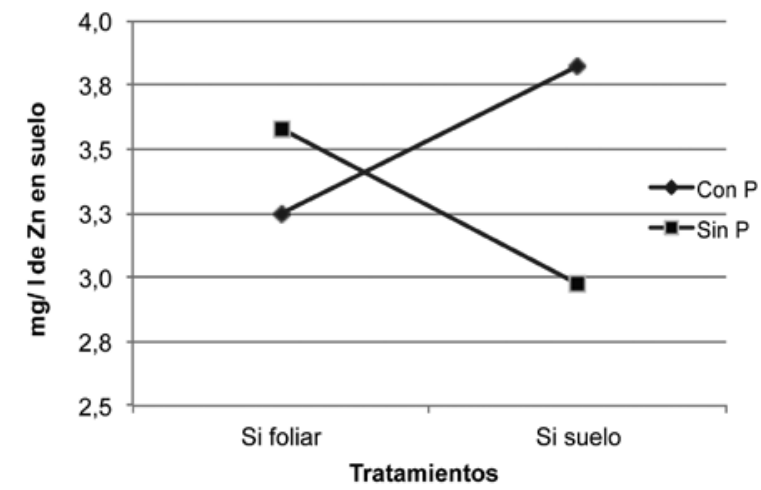

Figura 2. Comportamiento del zinc en el suelo en los tratamientos silicio y aplicación o no de plaguicidas $(\mathrm{P})$ en la segunda cosecha de arroz. Santa Clara, San Carlos, Costa Rica. Octubre, 2011.

Si foliar: silicio aplicado al follaje, Si suelo: silicio aplicado al suelo.

silicio, potasio, magnesio, calcio, manganeso, hierro, entre otras. Sin embargo, entre los resultados del análisis del elemento $\mathrm{Si}$ en el suelo después de cada una de las cosechas hubo diferencia, cuyos valores en el 2011 superaron a los de 2010 (Cuadro 1), pero

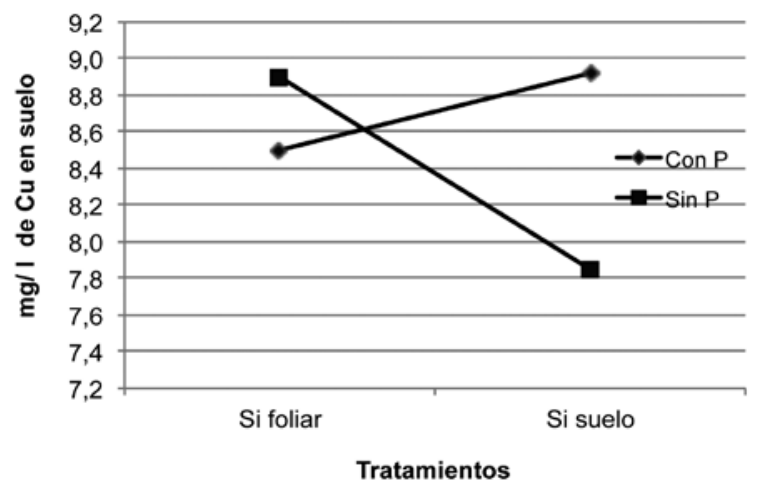

Figura 3. Comportamiento del cobre en el suelo en los tratamientos silicio y aplicación o no de plaguicidas $(\mathrm{P})$ en la segunda cosecha de arroz. Santa Clara, San Carlos, Costa Rica. Octubre 2011.

Si foliar: silicio aplicado al follaje, Si suelo: silicio aplicado al suelo.

este resultado no se tradujo en mejorías de las otras variables de suelo y de cultivo estudiado.

El comportamiento de los resultados entre tratamiento en el suelo es contrario a lo reportado y a lo propuesto por Quero (2008), Viana (2008b), Quero

Cuadro 1. Resultados de análisis de suelos previo a la siembra y posterior a las cosechas en cada tratamiento. Santa Clara, San Carlos, Costa Rica. Octubre de 2011.

\begin{tabular}{lcccccc}
\hline Variable & & $\mathbf{p H}$ & \multicolumn{2}{c}{ Acidez $(\mathbf{c m o l}(+) / \mathbf{l})$} & \multicolumn{2}{c}{ CICE $(\mathbf{c m o l}(+) / \mathbf{l})$} \\
\hline Año & $\mathbf{2 0 1 0}$ & $\mathbf{2 0 1 1}$ & $\mathbf{2 0 1 0}$ & $\mathbf{2 0 1 1}$ & $\mathbf{2 0 1 0}$ & $\mathbf{2 0 1 1}$ \\
\hline Previo siembra & 4,90 & & 1,20 & & 20,31 & \\
SFP & 4,68 & 4,85 & 1,58 & 1,35 & 22,87 & 22,50 \\
SF & 4,60 & 4,84 & 1,53 & 1,40 & 22,97 & 21,88 \\
SSP & 4,58 & 4,84 & 1,70 & 1,25 & 23,23 & 22,08 \\
SS & 4,73 & 4,88 & 1,60 & 1,23 & 22,22 & 22,56 \\
TT & 4,69 & 4,81 & 1,50 & 1,40 & 23,43 & 21,80 \\
\hline & $\mathbf{\% S a t u r a c i o ́ n ~} \mathbf{A l}$ & Suma bases $(\mathbf{c m o l}(+) / \mathbf{l})$ & \multicolumn{2}{c}{$\mathbf{S i}(\mathbf{m g} / \mathbf{l})$} \\
\hline Año & $\mathbf{2 0 1 0}$ & $\mathbf{2 0 1 1}$ & $\mathbf{2 0 1 0}$ & $\mathbf{2 0 1 1}$ & $\mathbf{2 0 1 0}$ & $\mathbf{2 0 1 1}$ \\
\hline Previo siembra & 5,91 & & 19,11 & & 44,7 & \\
SFP & 6,89 & 5,99 & 21,30 & 21,15 & 60,1 & 75,54 \\
SF & 6,64 & 6,43 & 21,44 & 20,48 & 41,67 & 51,88 \\
SSP & 7,32 & 5,66 & 21,53 & 20,83 & 41,24 & 72,35 \\
SS & 7,20 & 5,45 & 20,62 & 21,33 & 48,16 & 69,63 \\
TT & 6,40 & 6,43 & 21,93 & 20,40 & 49,72 & 60,76 \\
\hline
\end{tabular}

TT: tratamiento testigo, SS: silicio aplicado al suelo, SSP: silicio aplicado al suelo más plaguicidas, SF: silicio aplicado al follaje, SFP: silicio aplicado al follaje más plaguicidas. 
y Cárdenas (2006), Fertilizantes de Centroamérica (2004), entre otros autores, quienes indican que la aplicación de fuentes solubles de silicio al suelo mejora la fertilidad de este, en cuanto a la disminución del contenido de acidez intercambiable, incremento de la capacidad de intercambio catiónico, mayor disponibilidad del contenido de bases (calcio, magnesio, potasio), incremento de la solubilidad del fósforo que se encuentra fijado con el aluminio, hierro y manganeso en suelos con $\mathrm{pH}$ ácido. Para lograr estos beneficios, el suelo debe tener condiciones que cumplan con 100 a $200 \mathrm{mg} / \mathrm{l}$ de silicio soluble (Quero 2008), valores que son superiores al valor medio más alto $(75,5 \mathrm{mg} / \mathrm{l})$ encontrado en este estudio. En cambio, Vindas (2011) encontró respuesta positiva en la disminución de acidez intercambiable en suelos, después de dos años de aplicaciones de silicio cada mes en banano; en el caso del estudio que nos compete no se encontró ese resultado, con la diferencia que las aplicaciones se hicieron una vez cada año como normalmente lo hacen los productores de cultivos anuales, frecuencia de aplicación con la cual posiblemente no se alcancen los resultados deseados.

\section{Efecto de la aplicación de silicio en la concentración de nutrimentos en las hojas}

El contenido de manganeso a nivel de hojas en la siembra de 2010, mostró diferencia estadística significativa ( $\mathrm{p}$-valor $\leq 0,05$ ) en el tratamiento $\mathrm{SF}$ respecto a los demás (Figura 4). No obstante, todos los tratamientos tienen valores de manganeso dentro del rango considerado adecuado para el arroz, es decir, con poca probabilidad de ocasionar toxicidad para este cultivo por no superar $1000 \mathrm{mg} / \mathrm{kg}$ (Rodríguez 1999).

Al realizar los análisis estadísticos en el contenido de elementos nutritivos en las hojas, se encontró que sólo hubo diferencias significativas ( $\mathrm{p}$-valor $\leq 0,05$ ) entre tratamientos en el contenido de $\mathrm{Zn}$ en las hojas y en la interacción silicio-plaguicidas en el 2011 tanto en $\mathrm{Zn}$ como en $\mathrm{Mg}$, en la segunda siembra, con p-valor $=0,0012$ y 0,025 , respectivamente (Figuras 5,6 y 7). La prueba de Tuckey, indicó que el mejor tratamiento fue silicio al suelo con plaguicidas (SSP). Se observó que el $\mathrm{Zn}$ fue significativo tanto en suelo como en hojas, principalmente cuando se aplica el tratamiento silicio al suelo más plaguicidas al follaje y el tratamiento con silicio foliar solo, pero no se repite

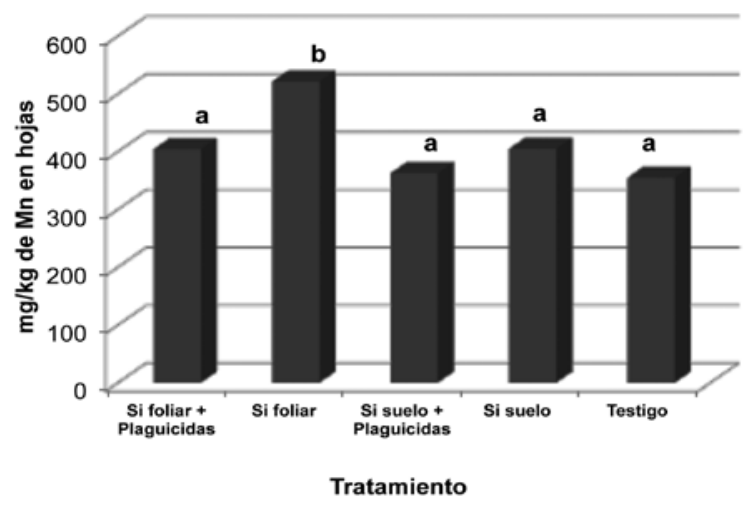

Figura 4. Representación de manganeso en las hojas de arroz en la cosecha del año 2010. Santa Clara, San Carlos, Costa Rica. Octubre, 2011.

el $\mathrm{Cu}$ a nivel de resultados en las hojas, como sucedió en el suelo.

La probabilidad de que los plaguicidas aplicados influyeran en el efecto de los tratamientos por contenido de $\mathrm{Zn}, \mathrm{Mg}$, $\mathrm{Mn}$ o $\mathrm{Cu}$ en estos, es baja, debido a que los ingredientes activos de estos insumos no sugieren la presencia de estos elementos. Del mismo modo, el muestreo para análisis foliar se hizo 45 días después y tres días antes de haber aplicado en todos los tratamientos multiminerales que, en sentido general, contienen $\mathrm{N}, \mathrm{P}, \mathrm{K}$, uno de ellos Ca y aminoácidos, pero no dispone de $\mathrm{Mg}$ ni de $\mathrm{Cu}$, otro contiene, además de $\mathrm{N}$ y $\mathrm{P}$, todos los microelementos; por las dos fechas de

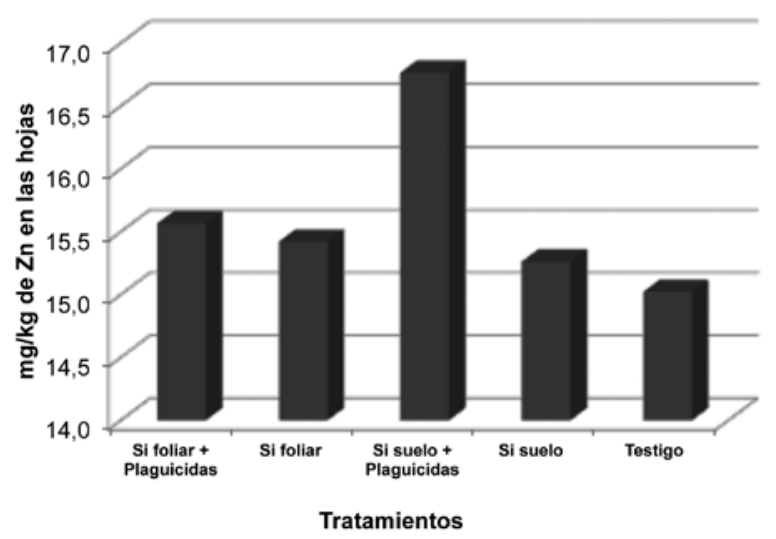

Figura 5. Representación de zinc en las hojas de arroz según tratamiento en la cosecha del año 2011. Santa Clara, San Carlos, Costa Rica. Octubre, 2011. 


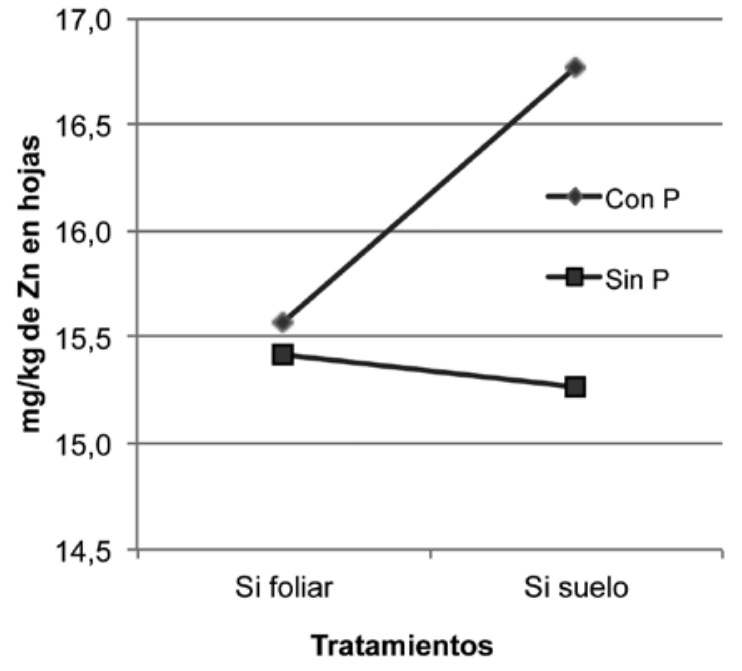

Figura 6. Comportamiento del zinc en las hojas en los tratamientos silicio y aplicación o no de plaguicidas $(\mathrm{P})$ en la segunda cosecha de arroz. Santa Clara, San Carlos, Costa Rica. Octubre, 2011.

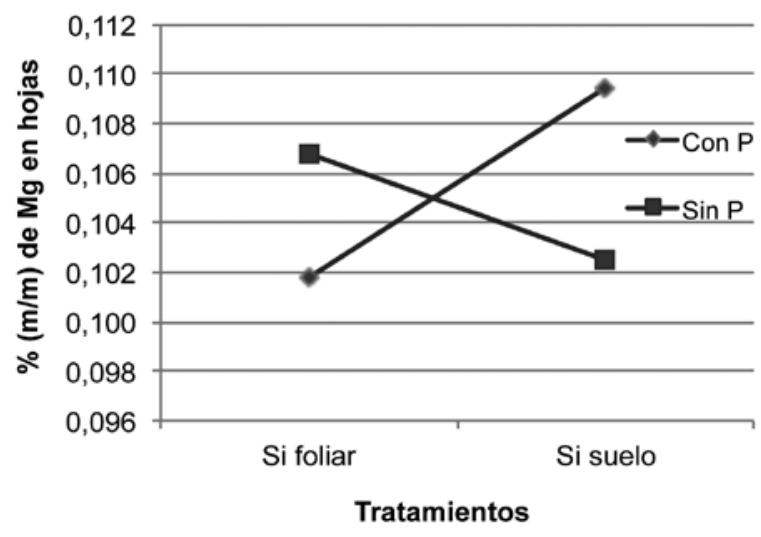

Figura 7. Comportamiento del magnesio en las hojas de arroz en los tratamientos silicio y aplicación o no de plaguicidas (P) en la segunda cosecha (2010). Santa Clara, San Carlos, Costa Rica. Octubre 2011.

aplicación la probabilidad que estos incidieran en el contenido de $\mathrm{Mn}, \mathrm{Zn}, \mathrm{Mg}$ y $\mathrm{Cu}$, es poca.

Se podría pensar que los plaguicidas aplicados al follaje llegaron al suelo, puesto que el tratamiento más fuerte en mostrar diferencias en contenido de $\mathrm{Zn}$ y $\mathrm{Cu}$ en suelo fue la aplicación de silicio al suelo más plaguicidas, cabe mencionar que las aplicaciones hechas con plaguicidas contenían un volumen de agua de 250 1/ha, cantidad de disolución con poca posibilidad de llegar al suelo en volumen importante.

Otra posibilidad que podría sugerir influencia sobre el $\mathrm{Zn}$ es la aplicación de fertilizante granular al suelo, durante el manejo del experimento en la segunda siembra se aplicó una fórmula con $1,05 \%$ de $\mathrm{Zn}$ a los 60 días después de la siembra (DDS), este $1,05 \%$ representa $1,57 \mathrm{~kg} / \mathrm{ha}$ del elemento al aplicar $150 \mathrm{~kg} / \mathrm{ha}$ de la fórmula, cantidad poco probable que apareciera en los resultados de los análisis foliares y de suelos hechos a los 90 DDS y posterior a la cosecha hecha a los 120 DDS.

El contenido de $\mathrm{Si}$ en las hojas no mostró diferencia significativa en los tratamientos tanto en 2010 como en 2011, quizás debido a la variabilidad de los resultados de los análisis en las unidades experimentales, con coeficientes de variación de 25,37 y 20,26 en hojas y 31,2 en suelo. Se encontró que en 2011 el contenido de $\mathrm{Si}$ en las hojas fue más alto en los tratamientos con silicio (Si): SS, SF y SSP, excepto SFP, respecto al testigo TT; mientras que en el 2010 fue más alto en todos los tratamientos al relacionarlo con el TT, manifestándose una inclinación hacia el mejoramiento del silicio en las hojas con la aplicación de este elemento.

A través de la prueba de $\mathrm{t}$, se encontró que los valores de Si en las hojas en el primer año (2010) fueron significativamente mayores que en la segunda siembra (Figura 8), resultados que deberían ser contrarios, mayores en las hojas en el 2011 después de haberse aplicado dos veces (en 2010 y 2011) en las mismas unidades experimentales.

Los resultados que muestran los valores próximos a 3\% de Si en las hojas en el 2010, son los más frecuentes y, en el 2011 el valor más frecuente es alrededor de $2 \%$. Estos datos no superan a los indicados por Winslow (1992), quien indica que el Si se acumula en el arroz en cantidades iguales o mayores al 5\%; Wild (1992) indica que la materia seca de la parte tallo-hojas del arroz alcanza entre 4 y $7 \%$ de $\mathrm{Si}$, y Mitani y Feng (2005), obtuvieron concentración de $7,3 \%$; la diferencia radica en que los valores indicados por estos autores se refieren a la parte de tallo y hojas en conjunto, mientras que los resultados del análisis en este estudio solo contempla las hojas.

Los elementos que mostraron diferencias significativas, tales como el $\mathrm{Zn}$ tanto en suelo como en hojas, el $\mathrm{Cu}$ en suelo, el $\mathrm{Mg}$ y el $\mathrm{Mn}$ en hojas, sugieren una tendencia a que los mejores tratamientos fueron $\mathrm{Si}$ al 


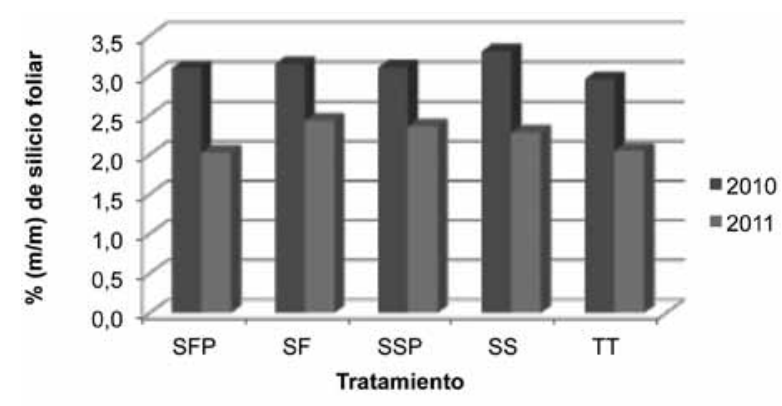

Figura 8. Representación de las diferencias del silicio en las hojas de arroz entre las dos cosechas, años 2010 y 2011. Octubre, 2011.

SFP: silicio aplicado al follaje más plaguicidas, SF: silicio aplicado al follaje, SSP: silicio aplicado al suelo más plaguicidas, SS: silicio aplicado al suelo, TT: tratamiento testigo.

suelo más plaguicidas (SSP) y Si foliar sin plaguicidas (SF). Lo anterior indica que el uso de Si foliar, mejora la absorción de estos elementos desde el suelo; por el contrario, al aplicar el tratamiento Si al suelo (SS) no mejoró la presencia de $\mathrm{Zn}, \mathrm{Cu}, \mathrm{Mg}$ y $\mathrm{Mn}$, pero sí cuando se combinó con aplicaciones de plaguicidas a nivel foliar.

Estos resultados sugieren que al parecer existe algún mecanismo de acción en conjunto entre la aplicación de Si al suelo y el uso de plaguicidas en el follaje. Este mecanismo puede ser debido a que los elementos del factor climático, mayormente lluvia y temperatura, que son importantes en esta zona, actúan sobre los minerales arcillosos del suelo liberando elementos nutricionales que en conjunto con el Si aplicado al suelo o natural, interactúen formando silicatos que pueden o no ser solubles, pero a su vez liberan otros elementos, que mejoran la disponibilidad de estos nutrimentos y la fertilidad en general del suelo (Quero 2008, Viana 2008b), este proceso ayudado por el posible control de plagas que ejercen los plaguicidas, podría coadyuvar a que el silicio aplicado se manifieste mejor. Por otro lado, la aplicación de silicio tiene acción sinérgica con el calcio, el magnesio, el potasio, entre otros elementos, mejorando el comportamiento de las plantas (Viana 2008b), posible razón del comportamiento del tratamiento Si aplicado a nivel foliar sin adicionar plaguicidas, pero que a la postre no se tradujo en mejoramiento de los rendimientos.

\section{Incidencia de enfermedades y plagas insectiles en el cultivo}

La aplicación y asimilación de Si tiende a reducir la incidencia y severidad de enfermedades e insectos en el cultivo del arroz (Datnoff et al. 1990, 1991, OsunaCanizales et al. 1991, Hernández 2002, Claudhary et al. 2003, Viana 2008a). Wild (1992) y Jones y Handreck (1967) asocian este comportamiento a que el silicio es absorbido por las plantas como ácido silícico, lo que hace suponer que el mecanismo de resistencia de la planta esté asociado a la cantidad de silicio en el tejido celular; igual idea presentan Winslow (1992) y Datnoff et al. (1997), dado que el Si se acumula en forma activa en el arroz con valor $\geq 5 \%$, lo que confiere crecimiento y resistencia

Esta resistencia aumenta directamente con el contenido de Si en la planta; sin embargo, en este experimento no se encontró diferencia estadística significativa a la aplicación de silicio tanto al suelo como a las hojas para la presencia de enfermedades, debido a la baja concentración de Si encontrada en las hojas en todos los tratamientos, la cual fue de alrededor de $3 \mathrm{y}$ $2 \%$ en los años 2010 y 2011, respectivamente. A pesar de la baja concentración de Si en las hojas, sin importar los tratamientos, se observó que el porcentaje promedio de incidencia de enfermedades fue muy bajo y similar en cada tratamiento, con un rango entre $11,01 \%$ encontrado en el tratamiento silicio aplicado al suelo más plaguicidas (SSP) en el tercer muestreo del año 2010 y $0,92 \%$ registrado en el mismo tratamiento, pero en el primer muestreo del año 2011. Del mismo modo, en varias unidades experimentales no se encontró larvas, pupas ni adultos en lo que se refiere a plagas. Es probable, que aunque las parcelas fueron relativamente grandes y limitadas una de otra para aislar el efecto de la deriva entre tratamientos con y sin plaguicidas, las condiciones de aplicación de plaguicidas en el entorno y manejo en general (producción de arroz comercial en la finca) afectaran la dinámica de las plagas y enfermedades, puesto que el comportamiento de la temperatura y distribución de las lluvias fueron similares a años anteriores con presencia de plagas.

Los análisis estadísticos realizados a la población de plantas muestreadas para incidencia de enfermedad no muestran diferencia estadística significativa $(\mathrm{p} \geq 0,05)$, lo que sugiere que hubo uniformidad en la 
población de plantas muestreadas para las variables de incidencia de enfermedad e insectos.

La variedad utilizada, CR 4477, es susceptible a Rhizoctonia sp., Pseudomonas sp. y Sarocladium sp., por otro lado muestra tolerancia alta a Pyricularia sp y cierta tolerancia a Helminthosporium sp. (ONS 2003), lo que concuerda con resultados de enfermedades encontrados donde en muestras tomadas en algunas unidades experimentales de los tratamientos y observadas en el microscopio, se encontró la presencia de Rhizoctonia sp. y en menor grado Helminthosporium sp., estos resultados al no ser obtenidos en cada una de las repeticiones de los tratamientos no fueron analizados estadísticamente, puesto que no era objetivo de este estudio especificar las enfermedades presentes, sino únicamente la incidencia de enfermedades.

\section{Componentes de rendimientos}

Los tallos totales se contabilizaron en un rango de 480 y 416 tallos $/ \mathrm{m}^{2}$, siendo el tratamiento SS el de mayor cantidad, mientras que el tratamiento con menor cantidad promedio fue SSP. En esta variable, igual que en tallos efectivos y porcentaje de granos vanos, no hubo diferencia significativa $(\mathrm{p} \leq 0,05)$. Los tallos efectivos fueron menores que $450 / \mathrm{m}^{2}$, valor considerado óptimo por Rivera (2011).

Respecto a la cantidad y longitud de panículas, se encontró que hubo diferencia estadística $(p \leq 0,05)$ entre tratamientos en la cosecha 2010 , en este caso el SS presentó la mayor cantidad de panículas de menor tamaño (categoría 1, es decir panículas pequeñas, con menos de $20 \mathrm{~cm}$ ); mientras que en la cosecha de 2011 hubo interacción silicio-plaguicidas (SSP) en las panículas categoría 1 (Figura 9), el tratamiento SF tuvo la mayor cantidad de panículas en esta categoría (Figuras 9 y 10). Cuando se aplicó plaguicidas la cantidad en esta categoría disminuyó, resultando favorable para el rendimiento, puesto que con la presencia de panículas de mayor longitud existe alta probabilidad de mayor rendimiento de granos. En las categorías de panícula con mayor longitud $(>20 \mathrm{~cm})$ no hubo diferencia estadística entre tratamientos, pero aquellos a los que se les aplicó plaguicidas tienen, por lo general, mayor porcentaje de panículas categoría 2 (Figura 10).

En la cosecha de 2010 al hacer los análisis estadístico incluyendo el testigo no hubo diferencias significativas, pero al analizar los tratamientos silicio con o sin plaguicidas hubo interacción silicio-plaguicidas $(\mathrm{p} \leq 0,05)$

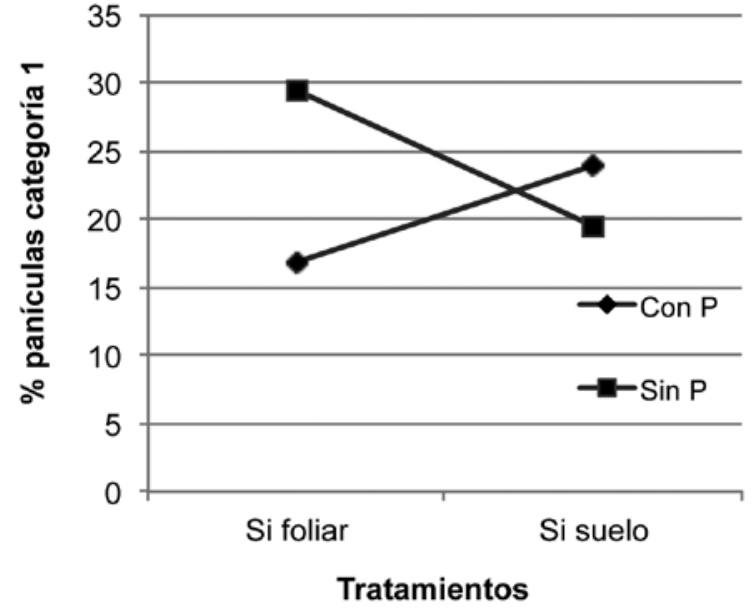

Figura 9. Comportamiento del porcentaje de panículas categoría 1 en los tratamientos silicio y aplicación o no de plaguicidas $(\mathrm{P})$ en la segunda cosecha de arroz. Santa Clara, San Carlos, Costa Rica. Octubre 2011.

para el peso de 1000 granos, siendo el tratamiento SF el mejor estadísticamente (Figura 11) con un promedio de 27,01 g y el menor peso de 24,41 g correspondió al tratamiento SS, pero en la cosecha de 2011, el tratamiento SF no mantuvo esa mejoría respecto a los demás tratamientos, al presentar el peso más bajo con $24,83 \mathrm{~g}$, estadísticamente significativo, por lo que se admite que el efecto por ese tratamiento no fue consistente entre una cosecha y la otra. Resultados como los obtenidos con las

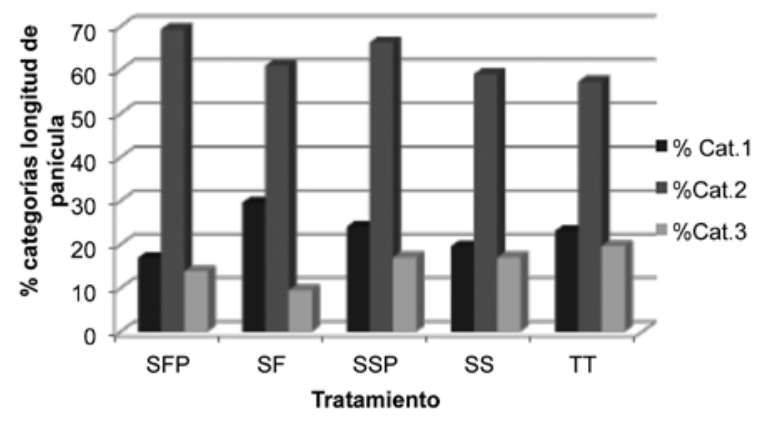

Figura 10. Representación del porcentaje de categorías de longitud de panículas en la segunda cosecha de arroz. Santa Clara, San Carlos, Costa Rica. Octubre 2011.

SFP: silicio aplicado al follaje más plaguicidas, SF: silicio aplicado al follaje, SSP: silicio aplicado al suelo más plaguicidas, SS: silicio aplicado al suelo, TT: tratamiento testigo.

AGRONOMÍA MESOAMERICANA 24(2):365-378. 2013 


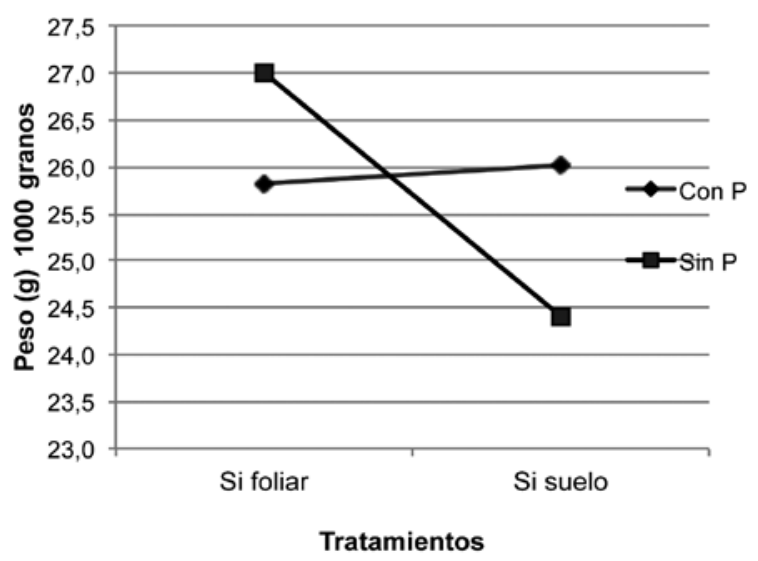

Figura 11. Comportamiento del peso de 1000 granos de arroz según tratamientos de silicio y aplicación o no de plaguicidas (P) en la primera cosecha (2010). Santa Clara, San Carlos, Costa Rica. Octubre 2011.

categorías de longitud de panículas en el tratamiento SS (mayor porcentaje de panículas pequeñas, pero menor en las categorías de longitud grande y relativo bajo peso (en 1000 granos), repercute en la producción final de granos, ya que son variables correlacionadas con el rendimiento. Sin embargo, los pesos de 1000 granos en todos los tratamientos fueron buenos respecto al valor de $24 \mathrm{~g}$ que presenta Rivera (2011) como suficiente.

En la cosecha de 2011 hubo diferencia estadística significativa $(\mathrm{p} \leq 0,05)$ cuando se aplicaron plaguicidas (promedio de 27,23 g/1000 granos) incluyendo el testigo, respecto a aquellos tratamientos donde no se hizo esta aplicación (Figura 12) (promedio de 25,39 g). La aplicación de silicio, sea al suelo o foliar refuerza en la planta su capacidad de almacenamiento y distribución de carbohidratos requeridos para el crecimiento y producción de cosecha (Quero 2008). Al parecer en este estudio, hubo un sinergismo entre el Si y con el uso de plaguicidas aplicados al follaje, quizás debido a la protección que ejercen estos sobre el cultivo que repercute en la producción de granos, dado que los mejores resultados fueron con silicio al suelo más plaguicida foliar.

\section{Rendimiento arroz en granza}

El rendimiento se presenta en peso húmedo y con impurezas, igual a como lo reporta la ONS (2003), con la diferencia que en este estudio la recolección se hizo manual, lo que pudo manifestarse en un rendimiento

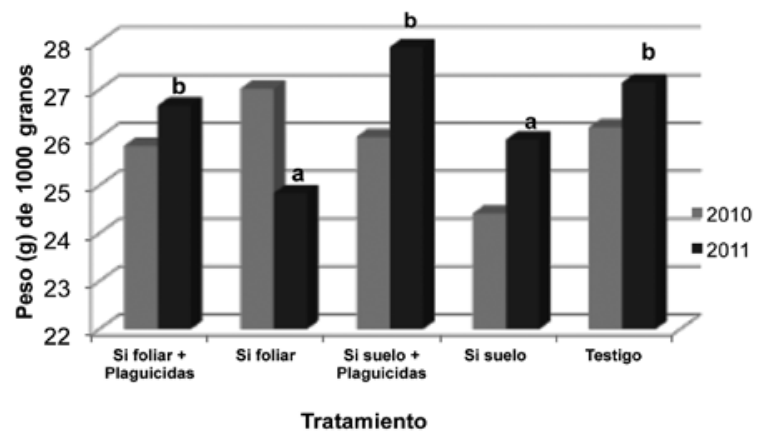

Figura 12. Representación del peso de 1000 granos de arroz en las dos cosechas. Santa Clara, San Carlos, Costa Rica. Octubre 2011.

mayor al obtenido a nivel nacional. En la cosecha del 2010 no hubo diferencia estadística significativa entre tratamientos para el rendimiento. Sin embargo, en la cosecha de 2011 hubo diferencia entre los tratamientos con plaguicidas (SFP, SSP, TT) y a aquellos que no se les aplicó (SS y SF) (Figura 13), igualmente entre los tratamientos Si más plaguicidas (SFP, SSP) frente SS y SF, es decir, excluyendo el testigo (TT). Tanto SF como SS, aunque con rendimientos de granos de arroz en granza buenos, respecto al promedio nacional (alrededor de 3,65 t/ha), fueron los más bajos con 5,91 y 6,29 t/ha, estos rendimientos altos se pudo deber a varias razones: la cosecha manual, son datos experimentales que por lo general tienen mejor manejo, la temperatura fue normal y las lluvias bien distribuidas lo que pudo repercutir en bajas incidencias de insectos $\mathrm{y}$ enfermedades. El tratamiento con mayor rendimiento fue SFP con 7,22 t/ha, a raíz de los resultados donde los mejores fueron aquellos cuando se combinaron la

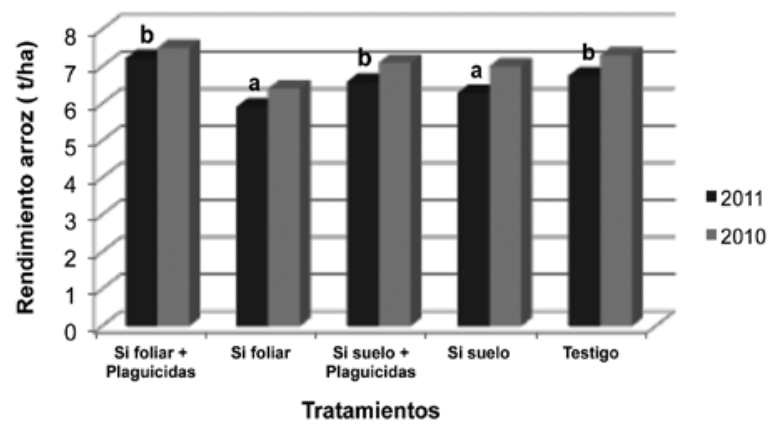

Figura 13. Representación del rendimiento de arroz en granza en las dos cosechas. Santa Clara, San Carlos, Costa Rica. Octubre, 2011. 
aplicación de silicio más el uso de plaguicidas, contrarios a los expuestos por Quero (2008), Viana (2008b) y Orejuela (2010). En cuanto a que el silicio por sí solo influye positivamente en la fertilidad de suelos, en el rendimiento del cultivo de arroz, en la disminución de incidencia de daños por insectos y enfermedades, se debería experimentar con fuentes de silicio en suelos más degradados que los presentes en este estudio y con mayor frecuencia de aplicación.

\section{Calidad de granos en granza}

No se encontraron diferencias significativas en las variables de calidad molinera, obtenidas por el Laboratorio de Control de Calidad de Arroz (Reforma del Decreto N³4487-MEIC-MAG-S RTCR 406-2007 2008, CONARROZ 2009), basado en el Reglamento Técnico de Arroz en Granza, documento "RTCR 4062007". En ninguno de los componentes de rendimiento de arroz pilado (\% de granos enteros, $\%$ quebrados gruesos, $\%$ de puntilla y $\%$ de semolina) hubo efecto del silicio, posiblemente debido a que, aunque los resultados de los análisis no mostraron altos contenidos de $\mathrm{Si}$ en el suelo y tenor de este en las hojas, no son suelos tan degradados para responder al Si como algunos sembrados de arroz en Brasil y Asia que responden positivamente a la aplicación de este elemento mineral (Fihlo et al. 2000, Kornörfer et al. 2002).
En la cosecha 2011, el rendimiento de semolina fue menor que la cosecha de 2010, por el contrario, el rendimiento de granos enteros y de puntilla fue mayor, lo que coincide con el mejor rendimiento de arroz en granza en la cosecha 2011; sin embargo en ambas cosechas la clasificación de la calidad fue baja (Cuadro 2).

Los componentes de calidad son bajos, estos son: \% de granos con 50\% o más de granos con apariencia a yeso, \% de granos dañados, \% de granos manchados, entre otros, que se usan para el pago al productor, según la caracterización del arroz en granza y los porcentajes establecidos para la base comercial del Ministerio de Economía, Industria y Comercio (Reforma del Decreto $\mathrm{N}^{\circ} 34487$-MEIC-MAG-S RTCR 406-2007 2008, CONARROZ 2009).

En el Cuadro 2 se presentan los valores de los componentes de calidad de arroz pilado, los mejores pagados son aquellos con mayor porcentaje de granos enteros y la mejor calidad se le confiere a los arroces que entran en la base dos o menos de grado de calidad, respecto al porcentaje de granos manchados, dañados, rojos y granos con alto porcentaje de apariencia a yeso establecido por el Ministerio de Economía, Industria y Comercio (Reforma del Decreto $\mathrm{N}^{\circ} 34487$-MEICMAG-S RTCR 406-2007 2008, CONARROZ 2009).

Se debe considerar que además del manejo del cultivo, las condiciones del clima al momento de la cosecha y el manejo poscosecha son de suma

Cuadro 2. Resultados de análisis de calidad de arroz de acuerdo a los Métodos de Análisis de Calidad de Arroz (MACA). CONARROZ. San José, Costa Rica. Diciembre 2011.

\begin{tabular}{|c|c|c|c|c|c|c|c|c|c|c|c|}
\hline \multirow[b]{3}{*}{$\begin{array}{l}\text { Componentes de } \\
\text { rendimiento }(\%)^{1}\end{array}$} & \multirow[b]{3}{*}{ Bases del MEIC* } & \multicolumn{5}{|c|}{2010} & \multicolumn{5}{|c|}{2011} \\
\hline & & \multicolumn{10}{|c|}{ Tratamientos } \\
\hline & & TT & SS & SSP & SF & SFP & TT & SS & SSP & SF & SFP \\
\hline RE & 52,264 & 48,2 & 48,7 & 51,3 & 49,8 & 49,7 & 54,6 & 52,4 & $\mathbf{5 4 , 9}$ & 48,8 & 54,3 \\
\hline RQG & 13,066 & 12,6 & 11,8 & 11,9 & 12,2 & 10,5 & 10,1 & 10,4 & 9,9 & 12,8 & 10,0 \\
\hline $\mathrm{RP}$ & 2,5 & 3,6 & 3,6 & 3,5 & 3,7 & 3,5 & 4,8 & 5,0 & $\mathbf{5 , 0}$ & 5,8 & 4,7 \\
\hline RS & 9 & 13,4 & 13,6 & 13,1 & 13,8 & 14,2 & 10,9 & 12,2 & 10,9 & 12,9 & 11,5 \\
\hline \multirow{2}{*}{$\begin{array}{l}\text { Variables grado } \\
\text { calidad }\end{array}$} & GD $(\%)$ & 2,8 & 2,7 & 2,3 & 2,2 & 2,8 & 4,7 & 2,0 & 3,2 & 1,8 & 7,7 \\
\hline & $\mathrm{GM}(\%)$ & 0,0 & 0,0 & 0,0 & 0,0 & 0,0 & 0,0 & 0,0 & 0,0 & 0,0 & 0,0 \\
\hline
\end{tabular}

${ }^{1}$ RE: rendimiento de granos enteros, RQG: rendimiento de granos quebrados gruesos, RP: rendimiento de puntilla, RS: rendimiento de semolina, GD: granos dañados, GM: granos manchados, TT: tratamiento testigo, SS: silicio aplicado al suelo, SSP: silicio aplicado al suelo más plaguicidas al follaje, SF: silicio aplicado al follaje, SFP: silicio aplicado al follaje más plaguicidas al follaje.

*Bases establecidas por el Ministerio de Economía de Industria y Comercio (MEIC). 
importancia en el rendimiento y calidad final del grano, inclinándose al manejo poscosecha el comportamiento de los resultados de calidad en este estudio.

\section{LITERATURA CITADA}

Almaguel, L; Botta E. 2005. Manejo integrado de Steneotarsonemus spinky, Smiley. Resultados de Cuba y transferencia para la región de Latinoamérica y el Caribe. In Curso de Postgrado de Acarología. Introducción a la Acarología Agrícola. Caso estudio: Steneotarsonemus spinky, Smiley 1967 (Tarsonemidae). La Habana, Cuba, INISAV. 37 p.

Balzarini, MG; Gonzalez, L; Tablada, M; Casanoves, F; Di Rienzo, JA; Robledo, CW. 2008. InfoStat, manual del usuario. Versión 2008. Córdoba, Argentina, Editorial Brujas. $331 \mathrm{p}$.

Chaudhary, RC; Nanda, JS; Tran, DV. 2003. Guía para identificar las limitaciones de campo en la producción de arroz. Problemas y limitaciones de la producción de arroz. Depósito de documentos de la FAO (Organización de las Naciones Unidas para la Agricultura y la Alimentación). Departamento de Agricultura. Roma 2003 (en línea). Consultado 29 set. 2008. Disponible en http://books.google.co.cr/ books?id=Xj_uqJ_by9MC\&printsec $=$ frontcover\# $\mathrm{v}=\mathrm{O}$ nepage \&q\&f=false

CONARROZ (Corporación Arrocera Nacional). 2009. Reglamento interno del mecanismo para la valoración del arroz en granza. San José, Costa Rica, Corporación Arrocera Nacional. 12 p.

CONARROZ (Corporación Nacional Arrocera). 2013. Estadísticas arroceras. Períodos 2010-2011 y 20112012. San José, Costa Rica. 2 p. (en línea). Consultado 29 jun. 2013. Disponible en http://www.conarroz.com/

Datnoff, LE; Raid, RN; Snyder, GH; Jones, DB. 1990. Evaluation of calcium silicate slag and nitrogen on brown spot, neck rot, and sheath blight development on rice. Biological and Cultural Tests for Control of Plant Disease 5:65.

Datnoff, LE; Raid, RN; Snyder, GH; Jones, DB. 1991. Effect of calcium silicate on blast and brown spot intensities and yields of rice. Plant Disease 75(7):729-732.

Datnoff, LE; Deren, CW; Snyder, GH. 1997. Silicon fertilization for disease management of rice in Florida. Crop Protection 16(6):525-531.
Fertilizante de Centroamérica S.A. 2004. El silicio en la agricultura. Informes técnicos (en línea). Consultado 15 dic. 2008. Disponible en webmaster@ fertica.com.pa.

Filho, BMP; Zinder, GH; Prabhu, AS; Datnoff, LE; Kornörfer, GH. 2000. Importancia do silicio para a cultura do arroz. Uma revisão de literatura. Potafos. Encarte Técnico 89. Informaciõnes agronõmicas 89-Março:8.

Hernández, GR. 2002. Nutrición mineral de las plantas. Facultad de Ciencias Forestales y Ambientales. Universidad de los Andes-Mérida, Venezuela (en línea). Consultado 11 dic. 2008. Disponible en www. forest.ula.ve/ rubenhg/nutricionmineral/

Infoagro (Sistema de Información del Sector Agropecuario Costarricense). 2010. Estadísticas agropecuarias. Estadísticas por actividad productiva. Arroz (en línea). Consultado 2 jun. 2010. Disponible en http://www. infoagro.go.cr.

Jones L, HP; Handreck, KA. 1967. Silica in soils, plants and animals. Advances in Agronomy (19):107-149.

Kornörfer, GH; Faria, RJ; Datnoff, LE; Pereira, LE. 2002. Influéncia do silicato do cálcium atolerência do arroz de sequeiro ao déficit hídrico do solo. In Reunião brasileira de fertilizante do solo e nutricão de plantas. Rio de Janeiro. Informancões agronõmicas $\mathrm{N}^{\mathrm{o}}$ 99-Septiembro/2002. Resumos. Brasil, SBCS. p. 16.

Mitani, N; Feng Ma, J. 2005. Uptake system of silicon in different plant species. Journal of Experimental Botany 56(414):1255-1261.

ONS (Oficina Nacional de Semillas). 2003. Características varietales y agronómicas de los materiales de arroz pertenecientes al registro de variedades comerciales (RVC) de la ONS. DOC 09-2003 D.T. San José, Costa Rica. 1 p.

Orejuela, J. 2010. Evaluación de la aplicación de varias dosis de ácido monosílico en la producción del cultivo de arroz. Var. INIAP 15. Guayaquil, EC. (en línea). Consultado 22 mayo 2010. Disponible en http://www.dspace.espol.edu. ec/bitstream/123456789/10892/3/OREJUELAS\%20 MAGALLANES\%20JUAN\%20DARIO.pdf

Osuna-Canizales, FJ; Dedatta, SK; Bonman, JM. 1991. Nitrogen form and silicon nutrition effects on resistance to blast disease of rice. Plant and Soil 135:223-231.

Quero, E. 2007. 12 virtudes del silicio. Biotecnología. Teorema ambiental. Revista Técnico Ambiental. Abril 2007. $N^{\circ}$ 63. Editorial 3W México S.A de CV (en línea). Consultado 29 sep. 2008. Disponible en http:// 
http://www.2000agro.com.mx/biotecnologia/12virtudes-del-silicio/

Quero, E. 2008. Silicio en la producción de chile. La biosilicificación proceso biológico fundamental en la productividad vegetal (en línea). Consultado 29 septiembre de 2008. Disponible en http://loquequero.com/portal/index. php?option=com_content $\&$ task=view $\& i d=20 \&$ Itemid $=1$

Quero, E; Cárdenas, C. 2006. Nueva tecnología para optimizar la producción agrícola tecnificada y tradicional y mejorar la calidad agrícola de los suelos. Instituto Tecnológico Superior de Uruapan. La Basilia, Uruapan, Michoacán México. 16 p.

Reforma del Decreto N³4487-MEIC-MAG-S RTCR 406-2007. 2008. Arroz en granza. Especificaciones y métodos de análisis para la comercialización e industrialización. San José, Costa Rica. La Gaceta $\mathrm{N}^{\circ} 205$ publicada en octubre de 2008. p. 4.

Rivera, CG. 2011. Manejo agronómico del cultivo de arroz (Oriza sativa) en Hacienda El Pelón De La Bajura, Liberia, Costa Rica. In Taller de Generación de Capacidades Vinculadas con el Sector Arrocero y el Cambio Climático. Comayagua, Honduras. p. 22. (en línea). Consultado 1 feb. 2013. Disponible en http:// curlacavunah.files.wordpress.com/2011/11/manejoagronomico-el-pelon-de-la-bajura.pdf
Rodríguez, JH. 1999. Fertilización del cultivo del arroz (Oryza sativa). In XI Congreso Nacional Agronómico/III Congreso Nacional de Suelos (en línea). Consultado 23 agosto 2011. Disponible en http://www.mag.go.cr/congreso_agronomico_xi/a506907-III_123.pdf

Viana, JE. 2008a. El silicio y la mosca blanca. Agromil. Tolima, Colombia (en línea). Consultado 15 sep. 2008. Disponible en http://www.silicioagromil.com.

Viana, JE. 2008b. Importancia del silicio en la nutrición vegetal. Agromil. Tolima, Colombia (en línea). Consultado 15 sep. 2008. Disponible en http://www. silicioagromil.com.

Vindas, FA. 2011. Efecto de la fertilización edáfica con dos fertilizantes silicatados sobre el $\mathrm{pH}$ y el aluminio intercambiable del suelo, la severidad de la sigatoka negra y la producción del banano (Musa AAA, cv. Grande Naine). Tesis de Lic., Costa Rica, Instituto Tecnológico de Costa Rica. 84 p.

Wild, A. 1992. Condiciones del suelo y desarrollo de las plantas según Russell. Madrid, España, Mundi-Prensa. $1045 \mathrm{p}$.

Winslow, MD. 1992. Silicon, disease resistance, and yield of rice genotypes under upland cultural conditions. Crop Science 32:1208-1213. 